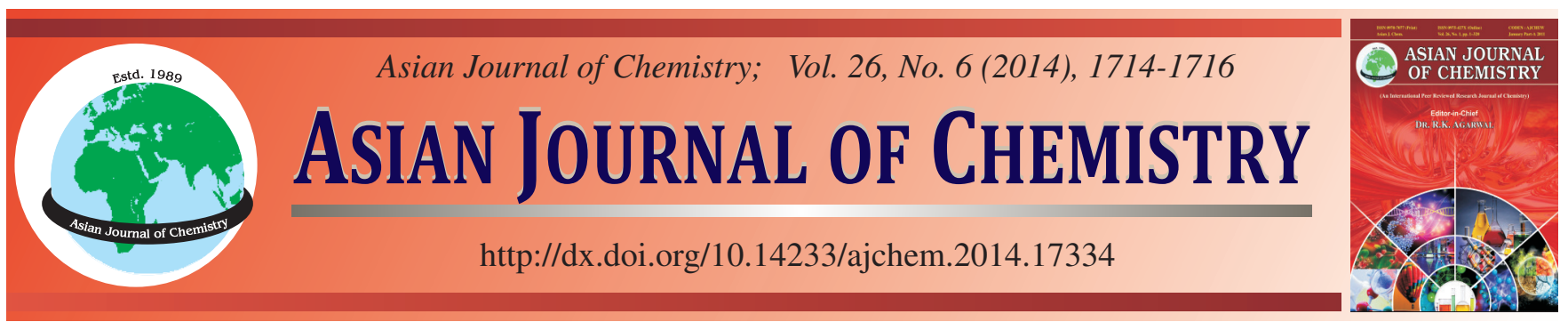

\title{
Tensile Mechanical Properties of Functional Gradient Hydroxyapatite/Polyether-ether-ketone Biocomposites $\dagger$
}

QIANQIAN SHEN and YusOng PAN*

Material Science and Engineering Institute, Anhui University of Science and Technology, Huainan 232001, Anhui Province, P.R. China

*Corresponding author: Tel: +86 15855419937; E-mail: yusongpan@163.com

The functional gradient hydroxyapatite/polyether-ether-ketone (HA/PEEK) biocomposites was fabricated by in situ synthesis method and incorporation with thermal pressure molding technology. The results of the tensile tests indicate that the tensile strength decreases gradually with the increase of hydroxyapatite content. The tensile strength of the functional gradient HA/PEEK biocomposites was improved after annealing treatment. The effect of annealing treatment on functional gradient HA/PEEK biocomposites gradually decreased with the rise of hydroxyapatite content.

Keywords: In situ synthesis, Thermal pressure molding technology, Hydroxyapatite, Polyether-ether-ketone, Tensile strength.

\section{INTRODUCTION}

Polyether-ether-ketone (PEEK) has already been used as biomaterials in the field of trauma, orthopedic and spinal implant ${ }^{1}$, due to their high temperature classification, radiation resistance, chemical resistance, high impact strength, good abrasion resistance and fatigue resistance. Hydroxyapatite (HA) is a major component of bone and teeth. Because of its high level of biocompatibility, hydroxyapatite has generally been used as a biological materials ${ }^{2,3}$. But the mechanical properties of hydroxyapatite is very brittle. The HA/PEEK composites is widely studied at present. The key is fabricate biological materials with high bioactivity and mechanical properties. In order to simultaneously optimize the bioactivity and mechanical properties, the functional gradient HA/PEEK (FG HA/PEEK) biocomposites were fabricated in this work.

\section{EXPERIMENTAL}

HA-PEEK mixture powder were produced containing $0-60 \mathrm{wt} \%$ hydroxyapatite by in situ synthesis ${ }^{4}$. The raw materials are calcium hydroxide, phosphoric acid and PEEK. The functional gradient HA/PEEK biocomposites was prepared with layer-by-layer method and incorporation with thermal pressure molding technology.

The schematic diagram of five layered FG HA/PEEK was shown in Fig. 1. The FG HA/PEEK biocomposites were com-

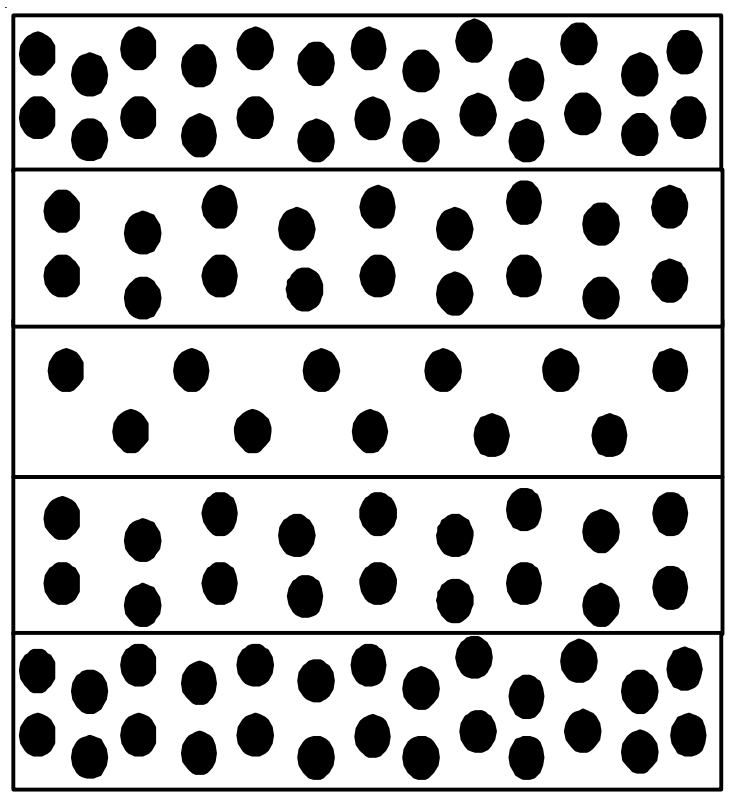

Fig. 1. Schematic diagram of five layered FG HA/PEEK biocomposites (denotation of hydroxyapatite particles; $\square$ denotation of PEEK matrix)

prised by five layers. Firstly, the HA-PEEK mixture powder with the highest content of hydroxyapatite was laid up ahead. The hydroxyapatite content gradually increased from interlayer

†Presented at The 7th International Conference on Multi-functional Materials and Applications, held on 22-24 November 2013, Anhui University of Science \& Technology, Huainan, Anhui Province, P.R. China 
to outer-layer. Secondly, FG HA/PEEK biocomposites were prepared using holding time in the range of 45-100 min at $375^{\circ} \mathrm{C}$ mold temperature with thermal pressure molding technology. The holding time increased, within that range, as the content of hydroxyapatite nano-powder reinforcement increased. According to this processing, the FG HA/PEEK biocomposites was fabricated. Some of the samples was annealed to study the effect of annealing treatment on the tensile strength and strain.

In this work, tensile tests were performed in accordance with WDW-50 with a crosshead speed of $1 \mathrm{~mm} / \mathrm{min}$. All the sample plates were cut into strips with the size of $110 \mathrm{~mm} \times$ $10 \mathrm{~mm} \times 3 \mathrm{~mm}$ for tensile tests. The same group average value was the experimental results.

\section{RESULTS AND DISCUSSION}

Change of tensile strength and strain with different contents of hydroxyapatite and annealing treatment: Fig. 2 shows the effects of different contents of hydroxyapatite reinforcement on the annealed and unannealed tensile strength and strain of FG HA/PEEK biocomposites. Fig. 2(a) suggested that the tensile strength of FG HA/PEEK biocomposites decreased with the rise of the hydroxyapatite content both before and after annealing treatment. The tensile strength decreased slowly, as the content of hydroxyapatite was higher than $10 \mathrm{wt} \%$ [Fig. 2(a)]. After annealing treatment the tensile strength is obviously higher than that of before annealing treatment. However, the effects of annealing treatment on
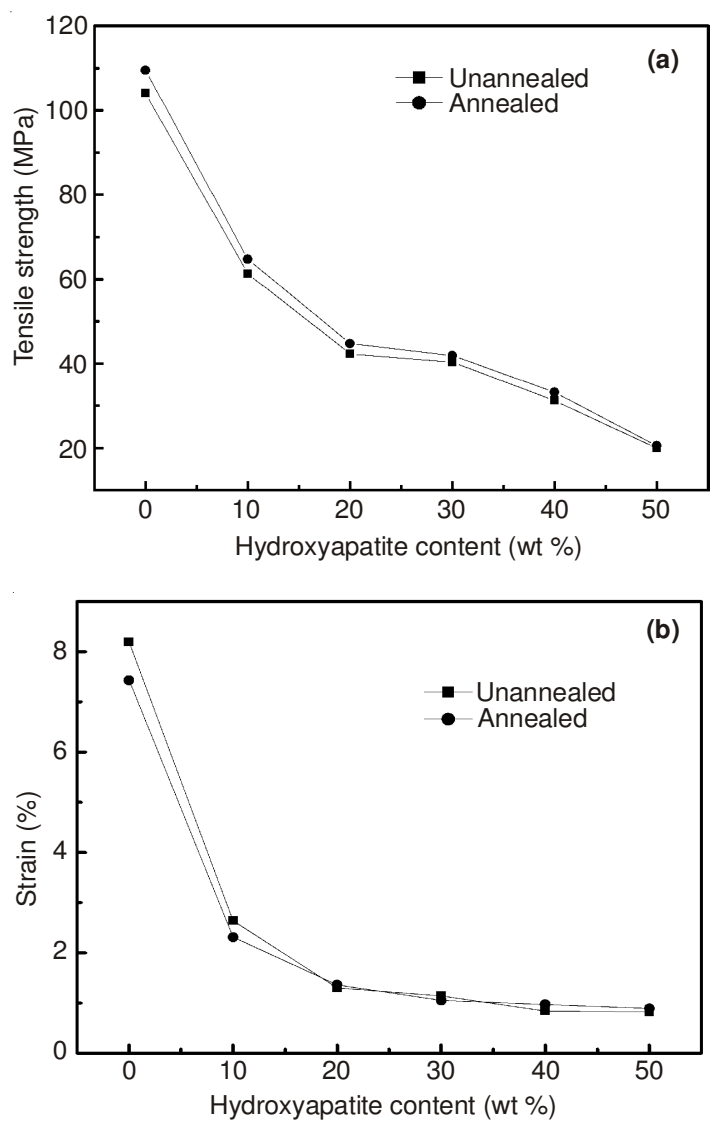

Fig. 2. Annealed and unannealed tensile strength (a) and strain (b) of nanoHA/PEEK FGM showing the effects of different levels of hydroxyapatite reinforcement at $10 \mathrm{HCDBAL}$ functional gradient HA/PEEK biocomposites gradually decreased with the rise of hydroxyapatite content [Fig. 2(a)]. The strain after annealing treatment is higher than before annealing treatment when hydroxyapatite content is lower than 10 wt \% [Fig. 2(b)]. When hydroxyapatite content is higher than $10 \mathrm{wt} \%$, there is no obvious influence of annealing treatment on the strain [Fig. 2(b)].

Fig. 3 shows the tensile strength-hydroxyapatite content and strain-hydroxyapatite content for tensile tests of annealed and unannealed FG HA/PEEK biocomposites reinforced with 20 and $30 \mathrm{wt} \%$ hydroxyapatite nano-powder. Tensile strength decreased with the content of hydroxyapatite increased before and after annealing treatment (Fig. 3(a)). However, the strain increased with the content of hydroxyapatite increased before annealing treatment and decreased with the increase of hydroxyapatite content after annealing treatment.
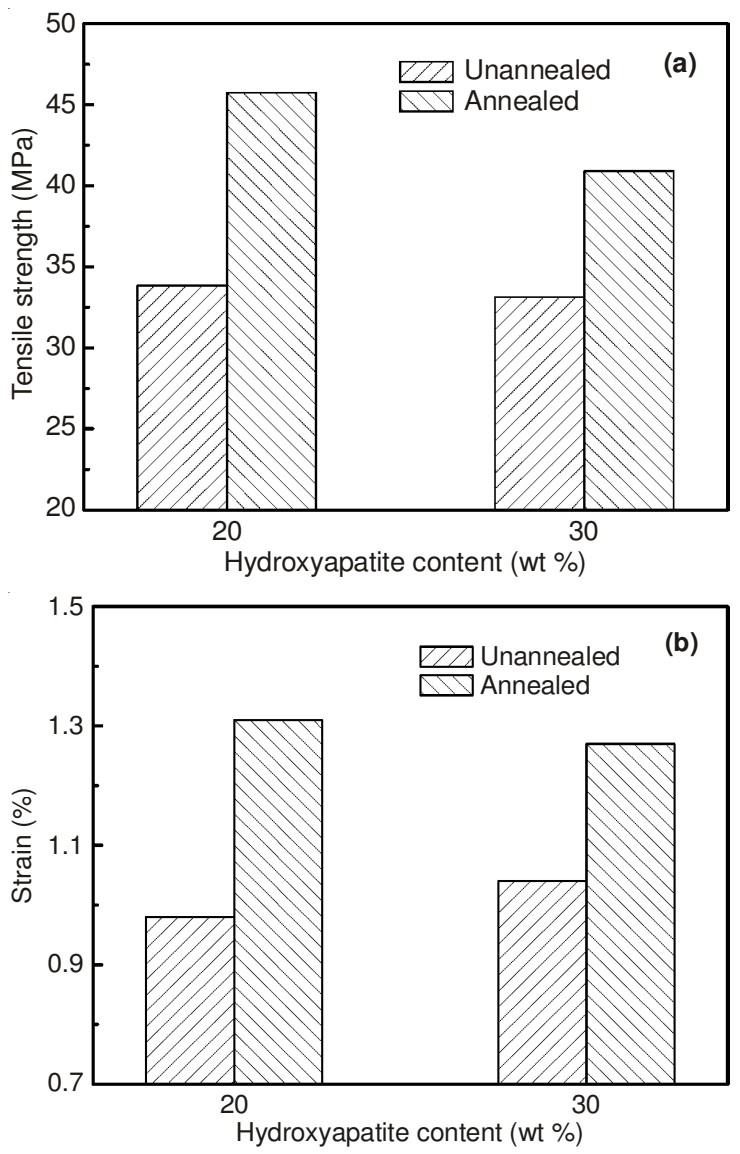

Fig. 3. Representative tensile strength-hydroxyapatite content(a) and strainhydroxyapatite content (b) for tensile tests of annealed and unannealed PEEK FGM reinforced with 20 and 30 wt \% hydroxyapatite nano-powder at $20 \mathrm{HCDBAL}$

All of these show that regardless of hydroxyapatite concentration difference between adjacent layers (HCDBAL) is 10 or 20, the tensile strength of FG HA/PEEK biocomposites decreased with the increase of the content of hydroxyapatite. There is no obvious change before and after annealing treatment when hydroxyapatite content higher than $10 \mathrm{wt} \%$.

Change of tensile strength with the HCDBAL: The effect of hydroxyapatite concentration difference between adjacent layers on the tensile strength is shown in Fig. 4. It can be concluded from Fig. 4 that the tensile strength of the 20,30 wt \% 


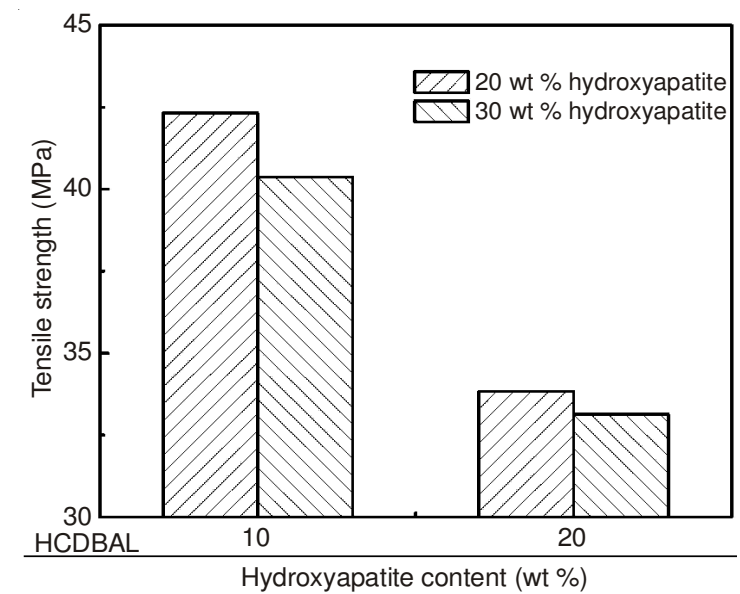

Fig. 4. Tensile strength of the unannealed PEEK functional gradient biocomposites reinforced with 20 and $30 \mathrm{wt}$. \% hydroxyapatite nano-powder at 10 and $20 \mathrm{HCDBAL}$

FG HA/PEEK biocomposites at $20 \mathrm{HCDBAL}$ is lower than 10 HCDBAL. This shows that the existence of the HCDBAL could increase the strength of the materials, but the higher HCDBAL would reduce the strength of the materials.

\section{Conclusion}

The tensile strength decreases gradually with the content of hydroxyapatite increased, while $10 \mathrm{wt} \%$ hydroxyapatite, has the highest strength $61.35 \mathrm{MPa}$ and strain $2.64 \%$. Annealing treatment can improve the tensile strength of the materials. The influence of annealing treatment on FG HA/PEEK biocomposites gradually decreased with the rise of hydroxyapatite content. When the HCDBAL is 10 , the tensile strength of the FG HA/PEEK biocomposites with same content of hydroxyapatite was improved.

\section{REFERENCES}

1. J.H. Lee, H.L. Jang, K.M. Lee, H.-R. Baek, K. Jin, K.S. Hong, J.H. Noh and H.-K. Lee, Acta Biomater., 9, 6177 (2013).

2. T.L. Conrad, D.J. Jaekel, S.M. Kurtz and R.K. Roeder, J. Biomed. Mater. Res. B Appl. Biomater., 101B, 576 (2013).

3. R. Ma, L.Q. Weng, X.J. Bao, Z. Ni, S. Song and W. Cai, Mater. Lett., 71, 117 (2012).

4. R. Ma, L.Q. Weng, X.J. Bao, S. Song and Y. Zhang, J. Appl. Polym. Sci., 127, 2581 (2013). 